\title{
MULTIPLIER PRESERVING ISOMORPHISMS BETWEEN MÖBIUS GROUPS
}

\author{
PEKKA TUKIA
}

Let us consider the case that we are given two groups $G$ and $G^{\prime}$ of conformal mappings acting in the unit disk $D=\{z \in \mathbf{C}:|z|<1\}$ such that $D / G$ and $D / G^{\prime}$ are Riemann surfaces. Let $\varphi: G \rightarrow G^{\prime}$ be an isomorphism. We would want to know under what circumstances $\varphi$ is a conjugation in a group $F$ containing at least all conformal self-mappings of $D$, i.e.

$$
\varphi(T)=f T f^{-1}, \quad T \in G, \quad f \in F .
$$

If the group $F$ is the group of all homeomorphisms of $F$, then $D / G$ and $D / G^{\prime}$ are homeomorphic (and $\varphi$ is induced by this homeomorphism); if $F$ is the group of all quasiconformal mappings of $D$, then $\varphi$ defines a point in the Teichmüller space of $G$; and finally if $F$ is the group of all Möbius transformations of $D$, then $\varphi$ defines the same point as id : $G \rightarrow G$ in the Teichmüller space of $G$.

The above problem in the theory of Riemann surfaces and Teichmüller spaces is our starting point in this paper. We generalize it in such a way that instead of the group of conformal mappings of the unit disk we consider the group of Möbius transformations of the $n$-sphere $S^{n}$. It also turns out that we need not demand that our groups act discontinuously; in fact a much weaker condition (concerning the existence of loxodromic mappings in the group) suffices. Under these conditions we prove that an isomorphism between two groups of Möbius transformations of $S^{n}$ is a conjugation by a Möbius transformation if and only if it has the property of "preserving multipliers" (Theorem 2). We also show that if such an isomorphism is a conjugation in the group of all quasiconformal mappings of $S^{n}$, then it cannot deform these "multipliers" arbitrarily: one can show that there is an upper limit to the deformation of multipliers (Theorem 1).

A Möbius transformation of $S^{n}$, the $n$-dimensional sphere, is a conformal or anticonformal self-map of $S^{n}$. Such mappings can be classified 
as loxodromic, parabolic or elliptic (if distinct from the identity) (see MartioSrebro [2], where a Möbius transformation was assumed to be orientation preserving, but the anticonformal case is not essentially different from that). If $S$ is a loxodromic Möbius transformation, it is conjugate in the group of all Möbius transformations of $S^{n}$ to a transformation of the form

$$
\begin{gathered}
T(z)=O(\lambda z)=\lambda O(z), \quad z \in \mathbf{R}^{n}, \quad 1<\lambda \in \mathbf{R}, \\
O \in O(n) \quad(T(\infty)=\infty),
\end{gathered}
$$

where $\mathrm{O}(n)$ is the group of orthogonal transformations of $\mathbf{R}^{n}$. We denote by $\mathrm{SO}(n)$ the group of orthogonal transformations of $\mathbf{R}^{n}$ with determinant 1. Then $T$ is orientation preserving if and only if $O \in \operatorname{SO}(n)$. The element $O \in \mathrm{O}(n)$ is not unique: only its conjugacy class depends on $S$ ( $=R T R^{-1}$ for some Möbius transformation $R$ ). In contrast, $\lambda$ is unique, supposing that $\infty$ is the attracting fixed point of $T$. This is equivalent to the fact that $\lambda>1$.

Henceforth we shall denote the attracting fixed point of a loxodromic transformation $S$ by $P(S)$; the repelling fixed point is $N(S)$. The group of all conformal and anticonformal Möbius transformations is denoted by $\operatorname{GM}(n)$; the subgroup consisting of all orientation preserving Möbius transformations is $\operatorname{SGM}(n)$. This does not seem to be the common usage: as a rule $\operatorname{GM}(n)$ is what we denote by $\operatorname{SGM}(n)$.

The real number $\lambda$ specified by (1) is called the multiplier of $S$ ( $=R T R^{-1}$ ) and denoted mul $S$. We denote by $\operatorname{rot} S$ the conjugacy class of $O$ in $\mathrm{O}(n)$. If $S$ is already in the form (1), i.e. it fixes 0 and $\infty$, we denote the element $O$ by $\operatorname{rot} S$. If $\operatorname{rot} S=\mathrm{id} \in \mathrm{O}(n)$ we say that $S$ is hyperbolic.

If a Möbius transformation $T$ of $S^{n}$ is not loxodromic it is elliptic or parabolic (if not the identity). It is elliptic if it (or its extension to $S^{n+1}$ ) can be put into the form (1) with $\lambda=1$ by conjugation. It is parabolic if it can be put into the form

$$
T(z)=O(z)+a, \quad \text { for } z \in \mathbf{R}^{n} \quad(T(\infty)=\infty),
$$

where $a \in \mathbb{R}^{n} \backslash\{0\}, O \in \mathrm{O}(n), O(a)=a$. If $T \in \mathrm{GM}(n)$ is not loxodromic we set mul $T=1$. If $T$ is parabolic we consider that the attracting and repelling fixed points of $T$ are defined and set $P(T)=N(T)=$ the fixed point of $T$. If $T$ is elliptic or the identity, $P(T)$ and $N(T)$ are not defined.

Let $G$ and $G^{\prime}$ be two subgroups of $\operatorname{GM}(n)$ and let

$$
\varphi: G \rightarrow G^{\prime}
$$

be an isomorphism with 


$$
\operatorname{mul} T=\operatorname{mul} \varphi(T)
$$

for every $T \in G$. Then we say that $\varphi$ is a multiplier preserving isomorphism.

Closely related to the concept of a multiplier preserving isomorphism is that of the dilatation of an isomorphism (cf. Sorvali [5]). Suppose that there is a real number $\mathrm{k} \geq 1$ such that, given an isomorphism $\varphi: G \rightarrow G^{\prime}$,

$$
(\operatorname{mul} T)^{1 / k} \leq \operatorname{mul} \varphi(T) \leq(\operatorname{mul} T)^{k},
$$

for every $T \in G$. Then we say that the dilatation of $\varphi$ is less than or equal to $k$. The dilatation of $\varphi$ is the smallest number $k$ for which these inequalities are valid. Of course, the dilatation of an isomorphism need not be finite. It is seen that to say that $\varphi$ is multiplier preserving amounts to the same as to say that the dilatation of $\varphi$ is 1 . If $\varphi$ is conjugation in the group of conformal and anticonformal Möbius transformations, then the dilatation of $\varphi$ is 1 . We shall show that if trivial cases are excluded, then the converse is also true.

Next we prove that if $\varphi$ is a conjugation by a $K$-quasiconformal selfmap of $S^{n}$, then the dilatation of $\varphi$ is less than or equal to $K$. In view of the fact that 1-quasiconformal self-maps of $S^{n}$ are just the Möbius transformations of $S^{n}$ (see Mostow [3]) this generalizes the statement that conjugation by a Möbius transformation does not change multipliers.

T h e o r e m 1. Let $G$ and $G^{\prime}$ be groups of Möbius transformations of $S^{n}$ $(n>1)$ and let $f: S^{n} \rightarrow S^{n}$ be K-quasiconformal. If $G^{\prime}=f G f^{-1}$ and

$$
\varphi(T)=f \circ T \circ f^{-1}, \quad \text { for } T \in G,
$$

then the dilatation of $\varphi$ is less than or equal to $K$.

Proof. If $T \in G$ is loxodromic, $T^{\prime}=\varphi(T)$ is also loxodromic. We may assume that both $T$ and $T^{\prime}$ fix 0 and $\infty$ and are of the form

$$
\begin{aligned}
T(z) & =O(\lambda z), \quad z \in \mathbf{R}^{n}, \quad O \in \mathrm{O}(n), \quad 1<\lambda \in \mathbf{R}, \\
T^{\prime}(z) & =O^{\prime}\left(\lambda^{\prime} z\right), \quad z \in \mathbf{R}^{n}, \quad O^{\prime} \in \mathrm{O}(n), \quad 1<\lambda^{\prime} \in \mathbf{R},
\end{aligned}
$$

and we must show that

$$
(\log \lambda) / K \leq \log \lambda^{\prime} \leq(\log \lambda) K .
$$

Let $D_{n}$ be the shell

$$
D_{n}=\left\{z \in \mathbf{R}^{n}: 1 \leq|z| \leq \lambda^{n}\right\}
$$

and

$$
D_{n}^{\prime}=f\left(D_{n}\right) .
$$

Since $f$ is $K$-quasiconformal we have

$$
K^{-1} \bmod D_{n} \leq \bmod D_{n}^{\prime} \leq K \bmod D_{n},
$$


where $\bmod D_{n}$ is defined by means of the conformal capacity of a shell (see Mostow [3] p. 80). We make use of the following facts concerning the modulus of a shell:

(a) If $D^{\prime} \subset D$, then $\bmod D^{\prime} \leq \bmod D$.

(b) If $D_{a b}=\left\{z \in \mathbf{R}^{n}: a \leq|z| \leq b\right\}$, then $\bmod D_{a b}=\log (b / a)$.

Using (a) and (b) we have

$K \log \lambda^{n}=K \bmod D_{n} \geq \bmod D_{n}^{\prime} \geq \bmod D_{M, \lambda^{\prime n}{ }_{m}}=\log \left(\lambda^{\prime n} m / \mathbf{M}\right)$

where

$$
\begin{aligned}
& M=\max \left\{|f(x)|: x \in S^{n-1}\right\} \\
& m=\min \left\{|f(x)|: x \in S^{n-1}\right\},
\end{aligned}
$$

i.e.,

$$
K \log \lambda \geq \log \lambda^{\prime}+(1 / n) \log (m / M) .
$$

Since this is true for every $n \in \mathbf{N}$, we must have

$$
K \log \lambda \geq \log \lambda^{\prime}
$$

Similarly one shows

$$
K^{-1} \log \lambda \leq \log \lambda^{\prime} .
$$

Remark. If $n=1$ and $f$ is $k$-quasisymmetric (this implies that $f(\infty)=\infty$ and that $f$ is increasing) then one can show that the dilatation of $\varphi$ is not greater than $\log 2 / \log (1+1 / k)$ (cf. Sorvali [5]). Sorvali's conditions are too strict; he assumes that $G$ and $G^{\prime}$ are covering groups. In fact his proof is valid, without any change, also if this assumption is dropped.

Next we prove that if the dilatation of $\varphi$ is 1 then it is a conjugation in the group of all (conformal or anticonformal) Möbius transformations. For this we need the following lemmata.

$\mathrm{L}$ e $\mathrm{m} \mathrm{ma} \mathrm{1.} \mathrm{Let} Q \in \mathrm{SO}(n)$ be fixed. Then there is a $K$-quasiconformal mapping $f: \mathbf{R}^{n} \rightarrow \mathbf{R}^{n}$ such that

$$
\begin{aligned}
& f(z)=z, \quad|z| \leq 1, \\
& f(z)=Q(z), \quad|z| \geq r,
\end{aligned}
$$

where $1<r$ and where $K$ depends on $r$ in such a way that there is a constant $c_{Q}$ (depends on $Q$ ) such that, beginning from some fixed $r$,

$$
K \leq 1+c_{Q} / \log r .
$$


Moreover, we have

$$
c=\sup \left\{c_{Q}: Q \in \mathrm{SO}(n)\right\}<\infty \text { and } \lim _{Q \rightarrow \mathrm{id}} c_{Q}=0 .
$$

Proof. The linear space $\mathbf{R}^{n}$ has a representation as a direct sum (see Greub [1]),

$$
\mathbf{R}^{n}=E_{1} \oplus \ldots \oplus E_{k},
$$

where each $E_{i}$ is a one- or two-dimensional linear subspace of $\mathbf{R}^{n}$, each $E_{i}$ is invariant under $Q$, the mapping $Q \mid E_{i}$ is the identity if $E_{i}$ is onedimensional, and $Q \mid E_{i}$ is an orientation preserving rotation of $E_{i}$ if $E_{i}$ is two-dimensional. Let $E_{1}, \ldots, E_{l}$ be one-dimensional, $E_{l+1}, \ldots, E_{k}$ be two-dimensional and let $Q \mid E_{i}, l<i \leq k$, be the rotation through the angle $\theta_{i}$.

We define the mapping $f_{0}$ as follows. For $1 \leq|z| \leq r_{0}, r_{0}>1$ let

$$
f_{0}(z)=O(z) z
$$

where $O(z) \in \mathrm{SO}(n)$ is the orthogonal mapping for which

$O(z) \mid E_{i}=$ id,$\quad 1 \leq i \leq l$,

$O(z) \mid E_{i}=$ the rotation through the angle $\left((|z|-1) /\left(r_{0}-1\right)\right) \theta_{i}$,

$$
l<i \leq k \text {. }
$$

It is clear that $O(z)$ depends only on $r=|z|$. We compute the derivative $f_{0}^{\prime}$ :

$$
f_{0}^{\prime}(z) h=\left(O^{\prime}(z) h\right) z+O(z) h, \quad h \in \mathbf{R}^{n}, \quad 1<|z|<r_{0} .
$$

As we have already observed it is possible to regard $O$ as a function of $r$ only. We do this and denote it also by $O$, it being clear from the context whether $O$ is regarded as a function of $z\left(\in \mathbf{R}^{n}\right)$ or $r(\in \mathbf{R})$. We have

$$
\begin{aligned}
O^{\prime}(z) h & =\left(O^{\prime}(r) \circ d r / d z\right) h \\
& =O^{\prime}(r)\left(z^{0}, h\right), \quad z, h \in \mathbf{R}^{n}, \quad 1<|z|<r_{0},
\end{aligned}
$$

where $z^{0}=z /|z|$,

and where the dual space of $\mathbf{R}^{n}$ is identified with $\mathbf{R}^{n}$ via the usual inner product (, ) of $\mathbf{R}^{n}$.

Thus

$$
\begin{aligned}
f_{0}^{\prime}(z) h & =\left(\left(O^{\prime}(r) \circ d r / d z\right) h\right) z+O(z) h \\
& =r\left(O^{\prime}(r)\left(z^{0}, h\right)\right) z^{0}+O(z) h, \quad z, h \in \mathbf{R}^{n}, \quad 1<|z|<r_{0} .
\end{aligned}
$$

The function $f_{0}$ defined by (2) has continuous derivatives in the shell $\left\{z: 1 \leq|z| \leq r_{0}\right\}, f(z)=z$ if $|z|=1$ and $f(z)=Q(z)$ for $|z|=r_{0}$. 
Thus we see that there is such a function as specified by the lemma for this particular $r_{0}$. We must study the behaviour of $K$ as $r$ varies. To do this we choose another $r_{1}>1$ and define a function $f_{1}$ using $f_{0}$.

Let $r_{1}>1$ be arbitrary and set

$$
\alpha=\frac{\log r_{0}}{\log r_{1}} .
$$

Then $r_{1}^{\alpha}=r_{0}$. Further, we define

$$
\begin{aligned}
f_{1}(z) & =f_{0}\left(|z|^{\alpha} z^{0}\right)|z|^{1-\alpha}=O\left(|z|^{\alpha} z^{0}\right) z \\
& =O\left(r^{\alpha}\right) z \quad\left(\text { for } z \in \mathbf{R}^{n}, \quad 1 \leq|z| \leq r_{1}, \quad z^{0} \quad\right. \text { defined in eq. (4)). }
\end{aligned}
$$

Then

$$
\begin{aligned}
f_{1}^{\prime}(z) h & =\alpha r^{\alpha-1}\left(\left(O^{\prime}\left(r^{\alpha}\right) \circ d r / d z\right) h\right) z+O\left(r^{\alpha}\right) h \\
& =\alpha r^{\alpha}\left(O^{\prime}\left(r^{\alpha}\right)\left(z^{0}, h\right)\right) z^{0}+O\left(r^{\alpha}\right) h .
\end{aligned}
$$

Let || || denote the usual norm in the space of linear mappings between two linear spaces. Since the matrix function $O$ has continuous derivatives,

$$
\sup _{1 \leq r \leq r_{0}}\left\|O^{\prime}(r)\right\|=c_{1}<\infty
$$

and $c_{1} \rightarrow 0$ as the original orthogonal mapping $Q$ tends to the identity. We have

$$
\sup _{1 \leq r \leq r_{1}}\left\|\alpha r^{\alpha}\left(O^{\prime}\left(r^{\alpha}\right) \circ d r / d z\right)\right\|=\alpha c_{2}, \quad c_{2}<\infty,
$$

where the same remark applies to $c_{2}$ as to $c_{1}$.

Since $\alpha=\log r_{0} / \log r_{1}$,

$$
f_{1}^{\prime}(z)=O(z) \circ\left(\mathrm{id}+C(r) / \log r_{1}\right)
$$

where $C(r)$ is a linear mapping $\mathbf{R}^{n} \rightarrow \mathbf{R}^{n}$ depending on $r$ in such a way that

$$
\sup _{1 \leq r \leq r_{1}}\|C(r)\| \leq c_{3}<\infty
$$

where $c_{3}$ depends only on $r_{0}$ and $Q$ and $c_{3}$ tends to zero as $Q$ tends to the identity. But if the derivative of $f_{1}$ is of the form $(6)$ and $C(r)$ fulfils (7), then the dilatation $K$ of $f_{1}$ satisfies

$$
K \leq 1+c / \log r_{1}
$$

where $c \rightarrow 0$ if the original orthogonal transformation $Q$ tends to the identity. Thus we have proved Lemma 1.

L e m m 2. Let $S$ and $T$ be two loxodromic transformations of $S^{n}$. Then, if $P(S)=P(T),($ or $N(S)=N(T))$, 


$$
\operatorname{mul} S^{l} T^{m}=\left((\operatorname{mul} S)^{l}(\operatorname{mul} T)^{m}\right)^{ \pm 1},
$$

for $l, m \in \boldsymbol{Z}$ (the exponent \pm 1 is so chosen that the resulting number is greater than 1).

Proof. We can assume that $S$ is of the form

$$
S(z)=\lambda_{1} O_{1}(z) \quad \text { for } z \in \mathbf{R}^{n} \quad\left(O_{1} \in \mathrm{O}(n), \quad 1<\lambda_{1} \in \mathbf{R}\right),
$$

and that $T$ is of the form

$T(z)=\lambda_{2} O_{2}(z-a)+a \quad$ for $\quad z \in \mathbb{R}^{n} \quad\left(a \in \mathbf{R}^{n}, \quad O_{2} \in \mathrm{O}(n), \quad 1<\lambda_{2} \in \mathbf{R}\right)$.

Then we have

$$
S^{l} T^{m}(z)=\lambda_{1}^{l} \lambda_{2}^{m} O_{1}^{l}\left(O_{2}^{m}(z)\right)-\lambda_{1}^{l} \lambda_{2}^{m} O_{1}^{l}\left(O_{2}^{m}(a)\right)+\lambda_{1}^{l} O_{1}^{l}(a)
$$

for $z \in \mathbf{R}^{n}$, and the lemma follows.

$\mathrm{L}$ e $\mathrm{m} \mathrm{m}$ a 3. Let $G$ be a subgroup of $\operatorname{GM}(n)$ generated by two loxodromic transformations $S$ and $T$ of $S^{n}$ without common fixed points. Let $G^{\prime}$ be another subgroup of $\mathrm{GM}(n)$ and $\varphi: G \rightarrow G^{\prime}$ be a multiplier preserving isomorphism. Then $\varphi(S)$ and $\varphi(T)$ do not have common fixed points.

Proof. We assume that $S^{\prime}=\varphi(S)$ and $T^{\prime}=\varphi(T)$ have common fixed points and derive a contradiction from this. If this were the case, there would be, by Lemma 2, infinitely many $(l, m) \in \mathbf{Z} \times \mathbf{Z}$ such that

$$
\operatorname{mul} S^{l} T^{m} \in U
$$

where $U$ is a given neighbourhood of $1 \in \mathbf{R}$.

Since $S$ and $T$ do not have common fixed points, $G$ can be supposed to be Schottky-like. (We can replace $G$ by the group generated by $S^{l}$ and $T^{l}$ for large enough $l$.) This means that there are four disjoint closed balls $A, B, C, D \subset S^{n}$ such that

$$
P(S) \in A, \quad N(S) \in B, \quad P(T) \in C, \quad N(T) \in D
$$

and that $\left(S^{n} \backslash(A \cup B \cup C \cup D)\right) \cap R\left(S^{n} \backslash(A \cup B \cup C \cup D)\right)=\varnothing$ for $R \in G \backslash\{\mathrm{id}\}$. Then it is easy to see that

$$
S^{l} T^{m}(A) \subset A, \quad\left(S^{l} T^{m}\right)^{-1}(D) \subset D, \quad l, m>0,
$$

i.e. $S^{l} T^{m}$ is loxodromic with $P\left(S^{l} T^{m}\right) \in A, N\left(S^{l} T^{m}\right) \in D$. Moreover, there is a real number $k>1$ depending on $A$ and $D$ (but not on $l$ and $m$ ) such that

$$
\operatorname{mul} S^{l} T^{m}>k
$$

is valid for all $l, m>0$, as is easily seen. In the same manner one can see that mul $S^{l} T^{m},(l, m) \neq 0$, is bounded away from 1 . But this contradicts Lemma 2 if $\varphi(T)$ and $\varphi(S)$ have common fixed points and $\varphi$ preserves multipliers. 
$\mathrm{L}$ e $\mathrm{mm}$ a 4. Let $G$ and $G^{\prime}$ be two subgroups of $\operatorname{GM}(n)$ such that $G$ is generated by loxodromic $T$ and $S$ without common fixed points. If $\varphi: G \rightarrow G^{\prime}$ is multiplier preserving, then there is a Möbius transformation $R \in \operatorname{GM}(n)$ such that

$$
\begin{array}{ll}
R(P(T))=P(\varphi(T)), & R(N(T))=N(\varphi(T)), \\
R(P(S))=P(\varphi(S)), & R(N(S))=N(\varphi(S)) .
\end{array}
$$

Proof. We assume $n \geq 3$. (If $G \subset \operatorname{GM}(n)$, then $G \subset \operatorname{GM}(m)$ for $m>n$.) Let $T^{\prime}=\varphi(T), \quad S^{\prime}=\varphi(S)$. We may suppose that $S$ and $T$ are conformal; otherwise we replace $S$ and $T$ by $S^{2}$ and $T^{2}$. (We do this substitution also if $S^{\prime}$ or $T^{\prime}$ is anticonformal.) Further, we can suppose that $T$ and $T^{\prime}$ fix 0 and $\infty$ and that $S$ and $S^{\prime}$ fix points in $\mathbf{R}^{2}$. If $R$ is a loxodromic Möbius transformation we define $R_{h}$ to be the unique hyperbolic transformation with the same attracting and repelling fixed points as $R$ and for which

$$
\operatorname{mul} R_{h}=\operatorname{mul} R \text {. }
$$

Let $G^{m}=\left\langle T^{m}, S^{m}\right\rangle$ be the group generated by $T^{m}$ and $S^{m}$, and denote $G^{\prime m}=\left\langle T^{\prime m}, S^{\prime m}\right\rangle, G_{h}^{m}=\left\langle T_{h}^{m}, S_{h}^{m}\right\rangle, G_{h}^{\prime m}=\left\langle T_{h}^{\prime m}, S_{h}^{\prime m}\right\rangle$, and let $\varphi^{m}: G^{m} \rightarrow G_{h}^{m}$ and $\varphi^{\prime m}: G^{\prime m} \rightarrow G_{h}^{\prime m}$ be the mappings defined by

$$
T^{m} \mapsto T_{h}^{m}, \quad S^{m} \mapsto S_{h}^{m} \text { and } T^{\prime m} \mapsto T_{h}^{\prime m}, \quad S^{\prime m} \mapsto S_{h}^{\prime m} .
$$

We wish to obtain an estimate for the dilatation $K_{m}$ of $\varphi^{m}$ and $K_{m}^{\prime}$ of $\varphi^{\prime m}$. We show that, beginning from some $m$,

$$
\begin{gathered}
K_{m} \leq 1+c\left(\operatorname{rot} T^{m}, \operatorname{rot} S^{m}\right) / m=1+c_{m} / m, \\
K_{m}^{\prime} \leq 1+c^{\prime}\left(\operatorname{rot} T^{\prime m}, \operatorname{rot} S^{\prime m}\right) / m=1+c_{m}^{\prime} / m,
\end{gathered}
$$

where $c(O, P), O, P \in \mathrm{SO}(n)$, are bounded and $c(O, P) \rightarrow 0$ as $O, P \rightarrow$ id in $\mathrm{SO}(n)$. (Since $\operatorname{rot} T$ and $\operatorname{rot} S$ are determined only up to the conjugacy class in $\mathrm{SO}(n), \mathrm{c}(O, P)$ depends only on the conjugacy class of $O$ and $P$.) $A$ similar remark applies to $c^{\prime}(O, P)$.

To prove (8) we note that, for large $m, G_{m}$ is Schottky-like, i.e. there are closed disjoint $n$-balls of $S^{n}$, denoted by $A_{m}, B_{m}, C_{m}, D_{m}$, such that

$$
P(T) \in A_{m}, \quad N(T) \in B_{m}, \quad P(S) \in C_{m}, \quad N(S) \in D_{m},
$$

$F_{m}=\operatorname{cl}\left(S^{n} \backslash\left(A_{m} \cup B_{m} \cup C_{m} \cup D_{m}\right)\right)$ being a fundamental domain for $G^{m}$. It is clear, at least for large $m$, that the balls can be so chosen that $F_{m}$ is also a fundamental domain for $G_{h}^{m}$. Schottky-groups are free, and so $G^{m}$ and $G_{h}^{m}$ are free, hence $\varphi^{m}$ is an isomorphism. Since $T$ is normalized to fix 0 and $\infty$, we can assume that $B_{m}$ is the unit ball $S^{n-1} \subset \mathbf{R}^{n}$ and that $A_{m}=(\operatorname{mul} T)^{m} B_{m}$. 
Now we can use Lemma 1 to find a $K$-quasiconformal self-mapping $f$ of the set $\left\{x \in \mathbf{R}^{n}: 1 \leq|x| \leq(\operatorname{mul} T)^{k+m}\right\}$, where $m, k \in \mathbf{Z}, m$ is large and fixed and $k>0$ varies, such that the following conditions are fulfilled:

(i) $f \mid\left\{x \in \mathbf{R}^{n}: 1 \leq|x| \leq(\operatorname{mul} T)^{m}\right\}=$ id,

(ii) $f\left|(\operatorname{mul} T)^{k+m} S^{n-1}=\operatorname{rot} T^{k+m}\right|(\operatorname{mul} T)^{k+m} S^{n-1}$,

(iii) $K \leq 1+c /(k \log (\operatorname{mul} T))$,

where $c$ depends on $\operatorname{rot} T^{k+m}$ in such a way that it tends to 0 as $\operatorname{rot} T^{k+m}$ tends to the identity in $\mathrm{SO}(n)$. We fix $m$ so that $C_{l} \cup D_{l} \subset\left\{z \in \mathbf{R}^{n}\right.$ : $\left.1 \leq|z| \leq \operatorname{mul} T^{m}\right\}$ for every $l>m$.

Define

$$
F^{k}=\left\langle T_{h}^{k}, S^{k}\right\rangle, \quad k>0,
$$

and let $\psi^{k}: G^{k} \rightarrow F^{k}$ be defined by

$$
\psi^{k}\left(T^{k}\right)=T_{h}^{k}, \quad \psi^{k}\left(S^{k}\right)=S^{k} .
$$

Then $\psi^{k}$ are isomorphisms for large $k$. Let $k>0$. Now we define a homeomorphism $f^{\prime}: S^{n} \rightarrow S^{n}$,

$$
f^{\prime}(x)=\left(\psi^{m+k}\right)^{-1}(R)(f(R(x))),
$$

if $R \in F^{m+k}$ and $R(x) \in \operatorname{cl}\left(S^{n} \backslash\left(A_{m+k} \cup B_{m+k} \cup C_{m+k} \cup D_{m+k}\right)\right)$. Since a $(n-1)$-sphere is a removable singularity for quasiconformal mappings in $n$-dimensional space, $f^{\prime}$ is $K$-quasiconformal in the set where it is now defined, i.e. in the regular set of $F^{m+k}$. We can extend it also to the limit set of $F^{m+k}$. Notice that if $x$ is a point of the limit set of $F^{m+k}$, then there is a sequence of elements $T_{i} \in F^{m+k}, i>0$, and $n-1$ spheres $E_{i} \in\left\{\mathrm{bd} A_{m+k}, \quad\right.$ bd $B_{m+k}, \quad$ bd $C_{m+k}, \quad$ bd $\left.D_{m+k}\right\}, \quad i>0$, such that $\lim _{i \rightarrow \infty} \operatorname{diam} T_{i}\left(E_{i}\right)=0$ (in the spherical metric of $S^{n}$ ) and that $\left\{T_{i}\left(E_{i}\right)\right\}_{i>j}$ and $x$ are in the same component of $S^{n} \backslash T_{j}\left(E_{j}\right)$ for all $j$. Now it is easy to see that $f^{\prime}\left(T_{i}\left(E_{i}\right)\right)_{i>0}$ converges to a point $y \in S^{n}$. We set $f^{\prime}(x)=y$. Extended this way, $f^{\prime}$ is a homeomorphism that induces $\left(\psi^{m+k}\right)^{-1}$. We know that it is $K$-quasiconformal outside the limit set of $F^{m+k}$.

Let $\psi^{\prime k}: F^{k} \rightarrow G_{h}^{k}$ be defined by

$$
\psi^{\prime k}\left(T_{h}^{k}\right)=T_{h}^{k}, \quad \psi^{\prime k}\left(S^{k}\right)=S_{h}^{k} \quad \text { for } k \geq m .
$$

As above, we can show that there is a homeomorphism $f^{\prime \prime}: S^{n} \rightarrow S^{n}$ such that $f^{\prime \prime-1}$ induces $\psi^{\prime m+k}$ for $k>0$, and that $f^{\prime \prime}$ is $K^{\prime}$-quasiconformal outside the limit set of $G_{h}^{m+k}$ with

$$
K^{\prime}<1+c^{\prime} /(k \log (\operatorname{mul} S))
$$


where $c^{\prime} \rightarrow 0$ as $\operatorname{rot} S^{m+k} \rightarrow$ id. Thus $f^{\prime} \circ f^{\prime \prime}$ is $K K^{\prime}$-quasiconformal outside the limit set of $G_{h}^{m+k}$. But $G_{h}^{m+k}$ fixes $S^{2}$, and consequently its limit set is contained in $S^{2}$. But $S^{2}$ is a removable singularity in $S^{n}$. Thus $f^{\prime} \circ f^{\prime \prime}$ is $K K^{\prime}$-quasiconformal in the whole $S^{n}$. Since $\varphi^{m+k}$ is induced by $\left(f^{\prime} \circ f^{\prime \prime}\right)^{-1}$, the first of the equalities in (8) is seen to be valid. The other equality in (8) is proved in the same manner.

Since $T_{h}, S_{h}, T_{h}^{\prime}$ and $S_{h}^{\prime}$ are Möbius transformations of $\mathbf{R}^{2}$, they can be represented as matrices of $\mathrm{SL}(2, \mathbf{C})$. In view of the above normalization we have

$$
\begin{gathered}
T_{h}=T_{h}^{\prime}=\left(\begin{array}{ll}
\lambda & 0 \\
0 & \lambda^{-1}
\end{array}\right), \quad \lambda^{2}=\operatorname{mul} T=\operatorname{mul} T^{\prime}, \\
S_{h}=\left(\begin{array}{ll}
a & b \\
c & d
\end{array}\right), \quad a d-b c=1, a+d=\zeta+\zeta^{-1}, \zeta=(\operatorname{mul} S)^{1 / 2}, \\
S_{h}^{\prime}=\left(\begin{array}{ll}
a^{\prime} & b^{\prime} \\
c^{\prime} & d^{\prime}
\end{array}\right), \quad a^{\prime} d^{\prime}-b^{\prime} c^{\prime}=1, a^{\prime}+d^{\prime}=\zeta+\zeta^{-1} .
\end{gathered}
$$

In diagonalized form we have

(*) $\quad S_{h}=\left(\begin{array}{ll}e & f \\ g & h\end{array}\right)\left(\begin{array}{ll}\zeta & 0 \\ 0 & \zeta^{-1}\end{array}\right)\left(\begin{array}{rr}h & -f \\ -g & e\end{array}\right)=\left(\begin{array}{cc}e h \zeta-f g \zeta^{-1} & \cdot \\ \cdot & -f g \zeta+e h \zeta^{-1}\end{array}\right)$, $e h-f g=1$. Similarly, $S_{h}^{\prime}$ can be diagonalized by a matrix $\left(e^{\prime} f^{\prime} \mid g^{\prime} h^{\prime}\right)$ with the same diagonal matrix. We have

$$
T_{h}^{m} S_{h}^{m}=\left(\begin{array}{cc}
\lambda^{m} \zeta^{m} e h-\lambda^{m} \zeta^{-m} f g & \cdot \\
\cdot & -\lambda^{-m} \zeta^{m} f g+\lambda^{-m} \zeta^{-m} e h
\end{array}\right) .
$$

It follows from (8) that the mapping $\varphi_{h}^{m}: G_{h}^{m} \rightarrow G_{h}^{\prime m}, T_{h}^{m} \mapsto T_{h}^{{ }^{\prime} m}$, $S_{h}^{m} \mapsto S_{h}^{\prime} m$ has bounded dilatation for large $m$ with dilatation less than or equal to

$$
1+c_{m}^{\prime \prime} / m
$$

where $c_{m}^{\prime \prime} \leq M<\infty$ beginning from some $m$ and $c_{m}^{\prime \prime}$ is near 0 if $\operatorname{rot} T^{m}$ is near id and $\operatorname{rot} S^{m}$ is near id. On the other hand, the multiplier of an element of $\operatorname{SL}(2, \mathbf{C})$ is

$$
\operatorname{mul}\left(\begin{array}{ll}
i & j \\
k & l
\end{array}\right)=\left(|i+l|+o\left(\mid i+l^{-1}\right)\right)^{2}
$$

where $o(x)$ tends to 0 as $x$ tends to 0 . Thus

$\frac{1}{2} \log \operatorname{mul} T_{h}^{m} S_{h}^{m}=m \log \lambda+m \log \zeta+\log |e h|+o\left(\lambda^{-m}+\zeta^{-m}\right)$

$\frac{1}{2} \log \operatorname{mul} T_{h}^{\prime m} S_{h}^{\prime m}=m \log \lambda+m \log \zeta+\log \left|e^{\prime} h^{\prime}\right|+o\left(\lambda^{-m}+\zeta^{-m}\right)$. 
In view of (9) we have

$$
\begin{gathered}
m \log \lambda+m \log \zeta+\log |e h|+o\left(\lambda^{-m}+\zeta^{-m}\right) \\
\leq\left(1+c_{m}^{\prime \prime} / m\right)\left(m \log \lambda+m \log \zeta+\log \left|e^{\prime} h^{\prime}\right|+o\left(\lambda^{-m}+\zeta^{-m}\right)\right)
\end{gathered}
$$

or

$$
\begin{aligned}
\log |e h| \leq \log \left|e^{\prime} h^{\prime}\right| & +\left(c_{m}^{\prime \prime} / m\right) \log \left|e^{\prime} h^{\prime}\right| \\
& +c_{m}^{\prime \prime}(\log \lambda+\log \zeta)+o\left(\lambda^{-m}+\zeta^{-m}\right) .
\end{aligned}
$$

Since the group $\mathrm{O}(n)$ is compact, there are arbitrarily large values of $m$ such that $\operatorname{rot} T^{m}$ and $\operatorname{rot} S^{m}$ are arbitrarily near id $\in \mathrm{O}(n)$ and thus, given $m_{0}$, there is always $m>m_{0}$ such that $c_{m}^{\prime \prime}<1 / m_{0}$. Therefore we must have

$$
\log |e h| \leq \log \left|e^{\prime} h^{\prime}\right| .
$$

Since the reversed inequality is also valid,

$$
|e h|=\left|e^{\prime} h^{\prime}\right| \text {. }
$$

If we replace $T_{h}^{m} S_{h}^{m}$ by $T_{h}^{m} S_{h}^{-m}$, a similar argument shows that

$$
|f g|=\left|f^{\prime} g^{\prime}\right| \text {. }
$$

Since the matrices of $\operatorname{SL}(2, \mathbf{C})$ have determinant equal to 1 , $e h-f g=1$ and $e^{\prime} h^{\prime}-f^{\prime} g^{\prime}=1$. If we combine this with (10) and (11), we see that the triangle with vertices 0,1 and $e h$ is equilateral with the triangle with vertices $0,1, e^{\prime} h^{\prime}$. Since they have the common side $[0,1]$, we must have either

$$
e h=e^{\prime} h^{\prime} \text { and } f g=f^{\prime} g^{\prime}
$$

or, alternatively,

$$
e h=\overline{e^{\prime} h^{\prime}} \text { and } f g=\overline{f^{\prime} g^{\prime}},
$$

the bar - denoting the complex conjugation .

Suppose we have the case (12). Equation (*) for $S_{h}$ and a similar (omitted) equation for $S_{h}^{\prime}$ show that

$$
a=a^{\prime}, \quad d=d^{\prime},
$$

i.e., $S_{h}$ and $S_{h}^{\prime}$ have equal diagonal elements. If we conjugate $S_{h}^{\prime}$ by a diagonal $R \in \mathrm{SL}(2, \mathbf{C})$ we have

$$
R S_{h}^{\prime} R^{-1}=\left(\begin{array}{ll}
\varkappa & 0 \\
0 & x^{-1}
\end{array}\right)\left(\begin{array}{ll}
a^{\prime} & b^{\prime} \\
c^{\prime} & d^{\prime}
\end{array}\right)\left(\begin{array}{cc}
x^{-1} & 0 \\
0 & \varkappa
\end{array}\right)=\left(\begin{array}{cc}
a^{\prime} & x^{2} b^{\prime} \\
x^{-2} c^{\prime} & d^{\prime}
\end{array}\right) .
$$

There is always $x \in \mathbf{C}$ such that $x^{2} b=b^{\prime}$ and, consequently, as the determinant of the matrix is $1, \varkappa^{-2} c^{\prime}=c$. But then $S_{h}=R S_{h}^{\prime} R^{-1}$ 
and in this case certainly $P(S)=P\left(S_{h}\right)=R\left(P\left(S_{h}^{\prime}\right)\right)=R\left(P\left(S^{\prime}\right)\right)$, and $N(S)=N\left(\mathrm{~S}_{h}\right)=R\left(N\left(S_{h}^{\prime}\right)\right)=R\left(N\left(S^{\prime}\right)\right)$. Since $T$ and $T^{\prime}$ were normalized to fix 0 and $\infty$ and $R$ can be extended to a Möbius transformation of $S^{n}$, the lemma follows in case (12).

In case (13) we denote by $R$ an element of $\mathrm{GM}(n)$ whose restriction to $\mathrm{S}^{2}$ is the complex conjugation $z \mapsto \bar{z}$. Then conjugation by $R$ reduces this case to the above case.

$\mathrm{T}$ h e o r e m 2. Let $G$ and $G^{\prime}$ be two groups of Möbius transformations of $S^{n}$ such that $G$ has at least two loxodromic transformations without common fixed points. Let $\varphi: G \rightarrow G^{\prime}$ be a multiplier preserving isomorphism. Further, suppose that

Fix $G=\{P(T): T \in G$ loxodromic $\}$

is not contained in any $k$-sphere $S^{k}$ or its image $T\left(S^{k}\right)$ for $k<n$, $T \in \operatorname{GM}(n)$. Then $\varphi$ is a conjugation in the group of all (conformal and anticonformal) Möbius transformations of $S^{n}$.

Proof. By assumption, there are two loxodromic transformations $T$, $S \in G$ without common fixed points. We assume that $N(T)=0$, $P(T)=\infty$ and prove Theorem 2 step by step.

A. If $Q, R \in G$ are loxodromic and

$$
\{P(Q), N(Q)\} \cap\{P(R), N(R)\}=\varnothing,
$$

then $\lim _{n \rightarrow \infty} P\left(Q^{n} R^{-n}\right)=P(Q)$ and $\lim _{n \rightarrow \infty} N\left(Q^{n} R^{-n}\right)=P(R)$.

The proof of $\mathrm{A}$ is based on the fact that for large $n$ the group generated by $Q^{n}$ and $R^{n}$ is Schottky-like. It is exactly similar to the proof of the case where $Q, R \in \mathrm{SL}(2, \mathbf{R})$, presented in Tukia [6], p. 9 .

B. If $R \in G$ is loxodromic, then there is $Q \in G$ such that $R$ and $Q$ do not have common fixed points.

For in the set $\{P(T), N(T), P(S), N(S)\}$ there are at least two points that do not belong to $\{P(R), N(R)\}$. Then the result follows by A.

C. Suppose $R, Q \in G$ are loxodromic, $P(R) \neq P(Q)$. Then there is a sequence of loxodromic $T_{n} \in G$ such that, if $T_{n}^{\prime}=\varphi\left(T_{n}\right)$,

$$
\begin{aligned}
& \lim _{n \rightarrow \infty} P\left(T_{n}\right)=P(R), \quad \lim _{n \rightarrow \infty} P\left(T_{n}^{\prime}\right)=P\left(R^{\prime}\right)=P(\varphi(R)), \\
& \lim _{n \rightarrow \infty} N\left(T_{n}\right)=P(Q), \quad \lim _{n \rightarrow \infty} N\left(T_{n}^{\prime}\right)=P\left(Q^{\prime}\right)=P(\varphi(Q)) .
\end{aligned}
$$

If the set $\{P(R), N(R), P(Q), N(Q)\}$ contains four elements, C follows by $\mathrm{A}$ and by Lemma 3 . If it contains three or two elements, we can find by $\mathrm{A}$ and $\mathrm{B}$ a series of loxodromic elements $S_{n} \in G$ such that $\lim _{n \rightarrow \infty} P\left(S_{n}\right)=P(Q), \lim _{n \rightarrow \infty} N\left(S_{n}\right) \neq P(R)$ and $\neq N(R)=N(Q)$. Now we can form a double series $T_{n m}$ with a subseries $T_{n_{k} m_{k}}$ fulfilling our conditions. 
Note that $\mathrm{C}$ is of course valid also if $P(R)=P(Q)$, but we do not need this.

We can define a mapping $f_{\varphi}:$ Fix $G \rightarrow$ Fix $G^{\prime}$ as follows. Let $R \in G$ be loxodromic. Then we set

$$
f_{\varphi}(P(R))=P(\varphi(R)) .
$$

We must show that this does not depend on the choice of $R$. Let $P(Q)=P(R), Q \in G$ loxodromic. Then by Lemma $3, Q^{\prime}=\varphi(Q)$ and $R^{\prime}=\varphi(R)$ have common fixed points. To see that $P\left(R^{\prime}\right)=P\left(Q^{\prime}\right)$, we use the equation of Lemma 2. For then

$$
\operatorname{mul} R^{l} Q^{m}=\left((\operatorname{mul} R)^{l}(\operatorname{mul} Q)^{m}\right)^{ \pm 1}, \quad l, m \in \mathbf{Z} .
$$

If $P(R)=N(Q)$, we must replace $m$ by $-m$ in the right side of (14). Since $\varphi$ is multiplier preserving, we must have $P\left(R^{\prime}\right)=P\left(Q^{\prime}\right)$.

We denote $T^{\prime}=\varphi(T)$ and $S^{\prime}=\varphi(S)$, where $T$ and $S$ are the elements of $G$ defined in the beginning of the proof. We suppose that $T^{\prime}$ fixes 0 and $\infty$ with $P\left(T^{\prime}\right)=\infty$. Moreover we suppose that $P(S)=P\left(S^{\prime}\right)$. This can always be achieved by conjugation which leaves 0 and $\infty$ fixed.

Now we claim:

(i) $|P(R)|=\left|P\left(R^{\prime}\right)\right|$, for $R \in G$ loxodromic, $R^{\prime}=\varphi(R),|P(R)|<\infty$,

(ii) $(P(R), P(Q))=\left(P\left(R^{\prime}\right), P\left(Q^{\prime}\right)\right)$, for $R, Q \in G$ loxodromic

$$
R^{\prime}=\varphi(R), \quad Q^{\prime}=\varphi\left(Q^{\prime}\right), \quad(|P(R)|, \quad|P(Q)|<\infty) .
$$

We prove (i) first. Assume $P(R) \neq P(S)$. By $\mathrm{C}$ there is a sequence $T_{n}$ of loxodromic elements of $G$ such that

$$
\begin{gathered}
\lim _{n \rightarrow \infty} P\left(T_{n}\right)=P(R), \quad \lim _{n \rightarrow \infty} P\left(T_{n}^{\prime}\right)=P\left(R^{\prime}\right), \quad\left(T_{n}^{\prime}=\varphi\left(T_{n}\right)\right), \\
\lim _{n \rightarrow \infty} N\left(T_{n}\right)=P(S), \quad \lim _{n \rightarrow \infty} N\left(T_{n}^{\prime}\right)=P\left(S^{\prime}\right) .
\end{gathered}
$$

By Lemma 4 there are orthogonal linear mappings $O_{m} \in \mathrm{O}(n)$ and real numbers $\lambda_{m}>0, m>0$, such that

$$
\lambda_{m} O_{m}\left(P\left(T_{m}\right)\right)=P\left(T_{m}^{\prime}\right) \text { and } \lambda_{m} O_{m}\left(N\left(T_{m}\right)\right)=N\left(T_{m}^{\prime}\right) .
$$

The family $\left\{O_{m}\right\}, m>0$, is a normal family and therefore there is a subsequence $O_{m_{1}}, O_{m_{2}}, \ldots$ for which the limit $\lim _{i \rightarrow \infty} O_{m_{i}}$ is an orthogonal linear mapping. Since $|P(S)|=\left|P\left(S^{\prime}\right)\right|$, we must also have $\lim _{i \rightarrow \infty} \lambda_{i}=1$. Therefore $\left|P\left(R^{\prime}\right)\right|=\lim _{n \rightarrow \infty}\left|P\left(T_{n}^{\prime}\right)\right|=\lim _{i \rightarrow \infty} \lambda_{m_{i}} \mid O_{m_{i}}\left(P\left(T_{m_{i}}\right)|=| P(R) \mid\right.$.

The proof of (ii) is the same; we only replace $S$ by $Q$.

But if a mapping satisfies (i) and (ii) then it must be the restriction of an orthogonal mapping of $\mathbf{R}^{n}$, which, moreover, is unique, since Fix $G$ 
spans $\mathbf{R}^{n}$. For simplicity, we can now assume that this orthogonal transformation is the identity and thus Fix $G=$ Fix $G^{\prime}$ and the mapping $P(R) \mapsto P(\varphi(R))$ is the identity, $R \in G$ loxodromic.

The proof of the theorem can now be concluded. We show that $T=T^{\prime}$, the normalized loxodromic transformation of $G$. We have

$$
\begin{array}{cl}
T(z)=\lambda O(z), & z \in \mathbf{R}^{n}, \quad O \in \mathrm{O}(n), \quad \lambda=\operatorname{mul} T=\operatorname{mul} T^{\prime}, \\
T^{\prime}(z)=\lambda O^{\prime}(z), \quad z \in \mathbf{R}^{n}, & O^{\prime} \in \mathrm{O}(n) .
\end{array}
$$

Thus $T=T^{\prime}$ if $O=O^{\prime}$. But since $O \mid$ Fix $G=O^{\prime} \mid$ Fix $G$ and since Fix $G$ spans $\mathbf{R}^{n}, O=O^{\prime}$. It is clear that $R=\varphi(R)$ also for other loxodromic $R \in G$. Finally, if $R \in G$ is arbitrary, there is a loxodromic $Q \in G$ such that $R Q$ is loxodromic. This shows that $\varphi(R)=R$. This concludes the proof.

Some related theorems. Sorvali [5] has proved our theorem for $\mathrm{SL}(2, \mathbf{R}) /\{1,-1\}$. His theorem is stated only for discrete groups but the proof does not make use of the discreteness of the groups. Selberg [4] has also proved similar results for deformations of groups of $\mathrm{SL}(n, \mathbf{R})$, stated in terms of traces of matrices of $\operatorname{SL}(n, \mathbf{R})$. The group of Möbius transformations of $\mathrm{S}^{n}$ can be identified with $\mathrm{O}(1, n+1) /\{1,-1\}$ and in view of this we might ask what the relation is between the multiplier and the trace of an element of $\mathrm{O}(1, n+1)$ which is a subgroup of $\mathrm{SL}(n+2, \mathbf{R})$. For $\operatorname{SL}(2, \mathbf{R})$ we have $|\operatorname{tr} T|=(\operatorname{mul} T)^{1 / 2}+(\operatorname{mul} T)^{-1 / 2}$.

If $T \in \mathrm{O}(1, n+1)$ is loxodromic when regarded as a transformation of $S^{n}$, it can be conjugated to the form

$$
T=\left(\begin{array}{ll}
\Lambda & 0 \\
0 & 0
\end{array}\right)
$$

where $\Lambda$ is a real $2 \times 2$-matrix

$$
\Lambda=\frac{1}{2}\left(\begin{array}{ll}
\lambda+\lambda^{-1} & \lambda-\lambda^{-1} \\
\lambda-\lambda^{-1} & \lambda+\lambda^{-1}
\end{array}\right)
$$

and $O \in \mathrm{O}(n)$ and $\lambda=\operatorname{mul} T$ (Mostow [3]). It follows:

$$
\operatorname{mul} T=\lim _{n \rightarrow \infty}\left|\operatorname{tr} T^{n}\right|^{1 / n} .
$$

Thus Theorem 2 is also valid if we replace the words "multiplier preserving" by "trace-preserving". Finally, we remark that if the Möbius group of $S^{2}$ is identified with $\mathrm{SL}(2, \mathbf{C}) /\{1,-1\}$, then the right side of (15) gives the square root of mul $T$. Thus in this case also the property that an isomorphism between Möbius groups preserves multipliers can be replaced by the requirement that it preserves the absolute values of traces. 


\section{References}

[1] GREUB, W.: Linear Algebra. - Springer-Verlag, Berlin-Göttingen-Heidelberg, 1963.

[2] MarTio, O., and U. SREBro: Automorphic quasimeromorphic mappings in $R^{n}$. To appear in Acta Math.

[3] Mostow, G. D.: Quasi-conformal mappings in $n$-space and the rigidity of hyperbolic space forms. - Inst. Hautes Etudes Sci. Publ. Math. 34, 1968, 53-104.

[4] SElBERG, A.: On discontinuous groups in higher-dimensional symmetric spaces. - Published in Contributions to Function Theory, TATA Inst. of Fund. Res., Bombay, 1960, 147-164.

[5] Sorvali, T.: The boundary mapping induced by an isomorphism of covering groups. - Ann. Acad. Sci. Fenn. Ser. A I 526, 1972, 1-31.

[6] Tukia, P.: On discrete groups of the unit disk and their isomorphisms. - Ann. Acad. Sci. Fenn. Ser. A I 504, 1972, 1-45.

University of Helsinki

Department of Mathematics

SF-00100 Helsinki 10

Finland

Received 5 June 1975 